\title{
Fractura mandibular patológica por plasmocitoma intraóseo
}

\section{Pathological mandibular fracture due to intraosseous plasmacytoma}

\author{
Antunes Freitas D*, Vergara Hernández CI**, Díaz Caballero A***, Moreira G****
}

\section{RESUMEN}

Los tumores de células plasmáticas se subclasifican en: Plasmocitomas solitarios de hueso, mieloma múltiple y plasmocitoma extramedular. El $80 \%$ de estos últimos afecta la región de la cabeza y cuello surgiendo en el tejido blando del conducto respiratorio alto y, raras veces, en la cavidad bucal. La búsqueda de una enfermedad sistémica es importante es aquellos casos en los que se haga dicho diagnóstico. Se presenta el caso de un paciente, varón de 73 años de edad, residente en la localidad rural de Gran Mogol, en Minas Gerais (Brasil), La tomografía computarizada mostró hipodensidad y reabsorción osteolítica irregular en la base de la cresta alveolar mandibular izquierda. La lesión de medición 38,0×19,0×15,0 mm. En la TC se ve claramente la presencia de fractura patológica del lado izquierdo. La aspiración con aguja se realizó, donde se obtuvo sangre. Se realizó la biopsia incisional. El diagnóstico presuntivo fue ameloblastoma. La muestra de biopsia fue enviada para análisis anatomohistopatológica. La microscopía mostró células tumorales compuesto por células monomórficas, con núcleos ovalados, grandes periféricos, y excéntricos, nucléolos prominentes y citoplasma basófilo, rodeado por estroma escaso y la infiltración de hueso esponjoso. La prueba de orina se destinó específicamente a la proteína de Bence-Jones, que fue negativo. Las radiografías de cráneo y tórax fueron realizados y no mostró cambios. Luego completó un diagnóstico de Plasmocitoma solitario en la mandíbula. El paciente está sometido a radioterapia en el Servicio de Oncología de la Santa Casa de Misericordia. El paciente tuvo mucositis severa y cambios en el color facial como resultado de la radioterapia.

Palabras clave: Plasmocitoma óseo, mandíbula, fractura patológica.

\section{SUMMARY}

Plasma cell tumors are subclassified into: Solitary plasmacytoma of bone, multiple myeloma and extramedullary plasmacytoma. $80 \%$ of the latter affects the region of the head and neck soft tissue arising in the upper respiratory duct and rarely in the oral cavity. The search for a systemic disease is important in cases where the diagnosis is made. A case of a patient, a man of 73 years of age, residing in the rural village of Great Mogol, Minas Gerais (Brazil), Computed tomography showed irregular osteolytic hypodensity and resorption at the base of the left mandibular alveolar ridge. The lesion measuring $38.0 \mathrm{~mm} \times 19.0 \mathrm{~mm} \times 15.0 \mathrm{~mm}$. On CT clearly shows the presence of pathological fracture of the left side. Needle aspiration was performed, where blood was obtained. Incisional biopsy was performed. The presumptive diagnosis was ameloblastoma. The biopsy specimen was sent for analysis anatomohistopatológica. Microscopy showed tumor cells composed of

* Odontólogo UNICOR. Especialista en Bioética UFLA. Profesor titular Facultad de Odontología FUNORTE Brasil, Campus da Saúde,

** Odontóloga Universidad de Cartagena. Especialista en Patología y Cirugía Oral Universidad Javeriana. Profesor titular Tiempo Completo, Vicedecana Curricular de la Facultad de Odontología de la universidad de Cartagena. Facultad de Odontología.

*** Odontólogo Universidad de Cartagena. Periodoncista Universidad Javeriana. Maestría en Educación Universidad del Norte. Profesor titular U. de C. Facultad de Odontología Universidad de Cartagena Campus de la Salud Zaragocilla. Cartagena.

**** Odontóloga UNIMONTES. Maestría Patología Oral UFMG. Profesor titular Facultad de Odontología FUNORTE, Brasil, Campus da Saúde, Montes Claros. 
monomorphic cells with oval nuclei, large peripheral and eccentric, prominent nucleoli and basophilic cytoplasm, surrounded by scant stroma and infiltration of cancellous bone. The urine test was conducted specifically for the Bence-Jones protein, which was negative. The skull and chest radiographs were performed and showed no changes. He then completed a diagnosis of solitary plasmacytoma of the jaw. The patient is undergoing radiotherapy at the Oncology Department of Santa Casa de Misericordia. The patient had severe mucositis and changes in facial color as a result of radiotherapy.

Key words: Bone plasmacytoma, mandible, pathological fracture.

Fecha de recepción: 15 de septiembre de 2010.

Aceptado para publicación: 18 de octubre de 2010.

Antunes Freitas D, Vergara Hernández CI, Díaz Caballero A, Moreira G. Fractura mandibular patológica por plasmocitoma intraóseo. Av. Odontoestomatol 2012; 28 (3): 119-124.

\section{INTRODUCCIÓN}

La primera proliferación neoplásica de células plasmáticas fue descrita por Dalrymmple y Bence-Jones en 1846 y Rustizky en 1873 acuñó el término de mieloma múltiple para esta enfermedad (1). Las neoplasias malignas originadas a partir de los inmunocitos de la serie B (linfocitos-B y plasmocitos) abarcan:

a) Mieloma múltiple y plasmocitoma solitario.

b) Macroglobulinemia de Waldeström y otras gammapatías monoclonales y

c) Leucemia linfoide crónica y linfomas malignos (2).

Los plasmocitomas solitarios (PS) son proliferaciones localizadas de plasmocitos que pueden aparecer en cualquier lugar donde existan formaciones del sistema reticuloendotelial. Estos son tumores infrecuentes, que abarcan entre un 5-10\% de las neoplasias de células plasmáticas. Se clasifican en Plasmocitoma óseo solitario (POS) y plasmocitoma extramedular (PEM) (3). Los POS afectan sobre todo a los huesos largos y esqueleto axial, siendo rara su localización a nivel de cabeza y cuello; cuando ocurre esta localización las áreas premolar y molar de la mandíbula son las más frecuentemente afectadas (46) Mientras que los PEM se localizan en áreas submucosas (7) y entre un 80-90\% acontecen a nivel de cabeza y cuello, específicamente a nivel del tracto aerodigestivo superior, afectando con mayor frecuencia a fosas, senos paranasales y rinofaringe. La localización laríngea es excepcional, siendo la epiglotis la estructura más frecuentemente afectada y en raras ocasiones en las amígdalas (8-10).
El plasmocitoma solitario es un tumor maligno derivado de un solo clon de células $B$ más o menos diferenciados. La naturaleza solitaria lo distingue de un mieloma múltiple intramedular, por otra parte la forma excepcional de plasmocitoma extramedular múltiples que pueden llegar a todos los tejidos reticuloendoteliales. El plasmocitoma solitario representa el $0,4 \%$ de los tumores malignas de cabeza y cuello. Así circunscrita, el concepto de plasmocitoma solitario se compone de dos entidades: la forma o el hueso intramedular (90\%) y la forma extramedular (10\%) preferentemente de llegar a la submucosa del tracto superior Aéreo. La última evolución en el hueso el mieloma múltiple es común que la forma intramedular (más del 50\%), la forma extramedular rara (menos del 20\%) (11). La enfermedad es más frecuente en los hombres y la edad promedio de diagnóstico es de unos 60 años (6).

La etiología sigue siendo desconocida, pero algunas ocupaciones, la exposición a ciertos productos químicos, la irradiación sobredosis, virus y factores genéticos se han sugerido como factores etiológicos $(4,6,11)$. En la mayoría de los casos tienen antecedentes de enfermedad autoinmune (5).

Los síntomas sistémicos incluyen dolor óseo, fracturas patológicas, insuficiencia renal, hipercalcemia, pérdida de peso, anemia, trombocitopenia y neutropenia (12).

Dentro de las características clínicas se observan una mucosa intensamente eritematosa con lesiones papilomatosas, nodulares o cambios aterciopelados 
superficiales. Los síntomas incluyen dolor en la boca de larga duración, disfagia, ronquera persistente, y faringitis (5).

El descubrimiento clínico no tiene nada específico, solo se habla en términos de su topografía. Las radiografías estándares iniciales no son específicas revelando lesiones osteolíticas que invaden estructuras vecinas. Las deficiencias deben dar lugar a una imagen de equilibrio TDM objetiva del tumor sin ser capaz de hacer un diagnóstico preciso $(1,13)$. El diagnóstico de esta patología se establece por la confirmación histológica de una proliferación maligna de células plasmáticas, que se componen de estructuras poligonales o redondas que se encuentran dispersas en el estroma relativamente escaso. Las células neoplásicas además presentan un núcleo grande, excéntrico individual, similar a las células típicas de plasma. El diagnóstico además se basa en pruebas hematológicas y hallazgos bioquímicos, análisis de orina y radiografías del esqueleto $(6,8,14)$. El tratamiento consiste, principalmente la irradiación y la quimioterapia así como el uso de corticoides (2, $6,15)$ y el pronóstico es generalmente pobre $(6,12)$.

\section{PRESENTACIÓN DEL CASO CLÍNICO}

Paciente, varón de 73 años de edad, residente en la localidad rural de Gran Mogol, en Minas Gerais (Brasil), se presentó al dentista con diagnóstico clínico enviado por el Sistema de Salud Pública Municipal, por asimetría facial en el lado izquierda. Al examen clínico intraoral se aprecia una lesión en el maxilar inferior izquierdo que va desde la región premolar a la molar. El paciente refiere dolor, y afirma que esta primera hinchazón facial se inició hace 3 meses. El paciente es hipertenso y utiliza la medicación para ser controlado. Al paciente se le pidió llevar a cabo la tomografía de la mandíbula para evaluar la lesión. La tomografía computarizada mostró hipodensidad y reabsorción osteolítica irregular en la base de la cresta alveolar mandibular izquierda. La lesión de medición $38 \times 19 \times 15$ mm (Fig. 1). En la TC se ve claramente la presencia de fractura patológica del lado izquierdo (Fig. 2). La aspiración con aguja se realizó, donde se obtuvo sangre. Se realizó la biopsia incisional. El diagnóstico presuntivo fue ameloblastoma. La muestra de biopsia fue enviada para análi-

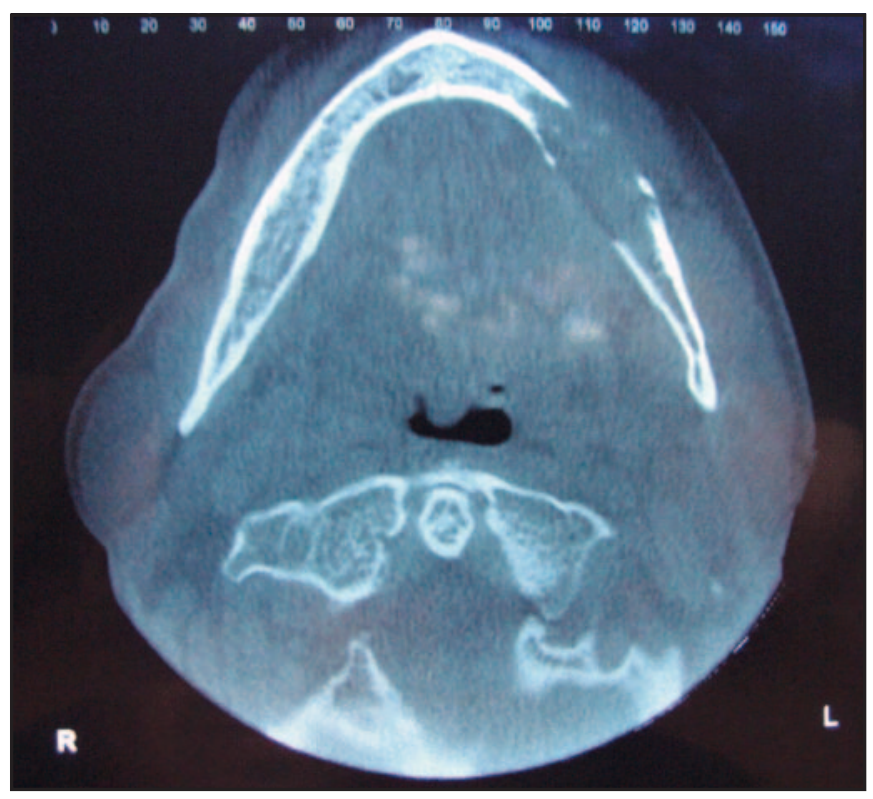

Fig. 1. Tomografía axial computarizada: hipodensidad y reabsorción osteolítica irregular en la base de la cresta alveolar mandibular izquierda.

sis anatomohistopatológica. La microscopía mostró células tumorales compuesto por células monomórficas, con núcleos ovalados, grandes periféricos, y excéntricos, nucléolos prominentes y citoplasma basófilo, rodeado por estroma escaso y la infiltración de hueso esponjoso Figs. 3 y 4). La prueba de orina se destinó específicamente a la proteína de BenceJones, que fue negativo. Las radiografías de cráneo y tórax fueron realizados y no mostró cambios (Figs. 5 y 6). Luego completó un diagnóstico de Plasmocitoma solitario en la mandíbula. El paciente está sometido a radioterapia en el Servicio de Oncología de la Santa Casa de Misericordia. El paciente tuvo mucositis severa (Fig. 7) y cambios en el color facial como resultado de la radioterapia (Fig. 8).

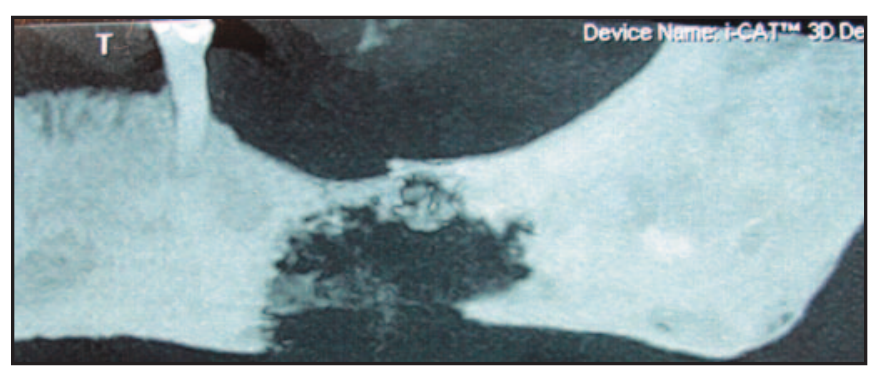

Fig. 2. Tac: Fractura cuerpo mandibular izquierda. 


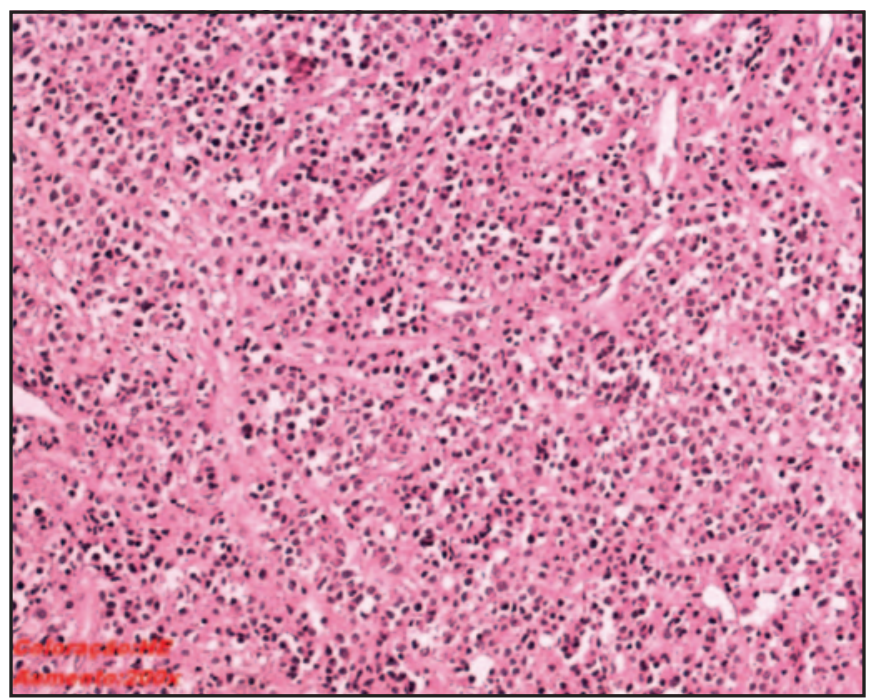

Fig. 3. Células tumorales compuesto por células monomórficas, con núcleos ovalados, grandes periféricos, y excéntricos, nucléolos prominentes y citoplasma basófilo. Aumento 200x.

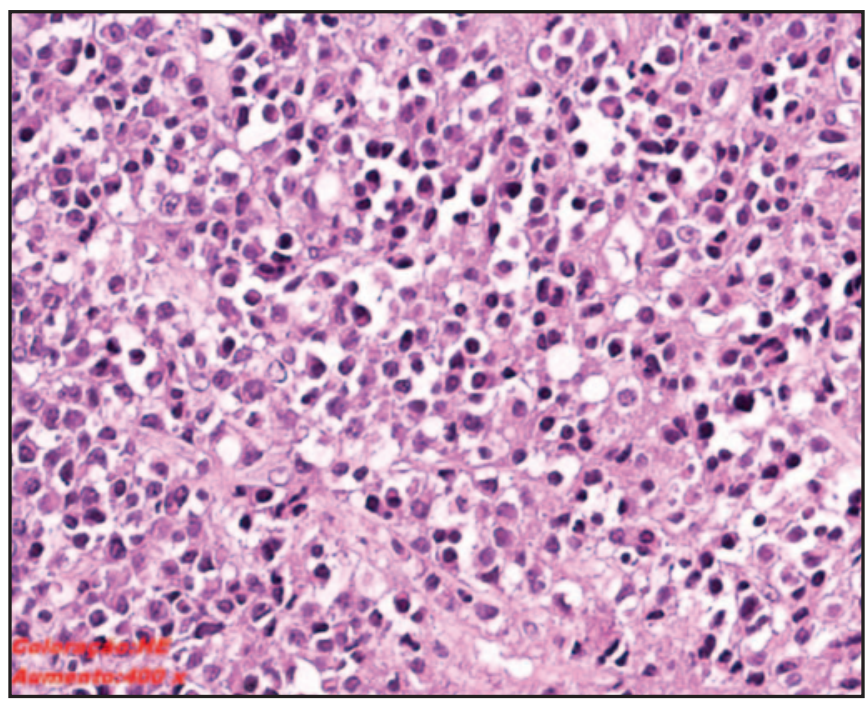

Fig. 4. Células tumorales compuesto por células monomórficas, con núcleos ovalados, grandes periféricos, y excéntricos, nucléolos prominentes y citoplasma basófilo. Aumento 400x.

\section{DISCUSIÓN}

La prevalencia del plasmocitoma intraóseo solitario en cabeza y cuello y particularmente en mandíbula es extremadamente baja de acuerdo a lo reportado en múltiples artículos relacionados con el tema $(4-6,12)$.

Dentro de los síntomas iniciales se encuentran el dolor y la fractura patológica a nivel del hueso afec-

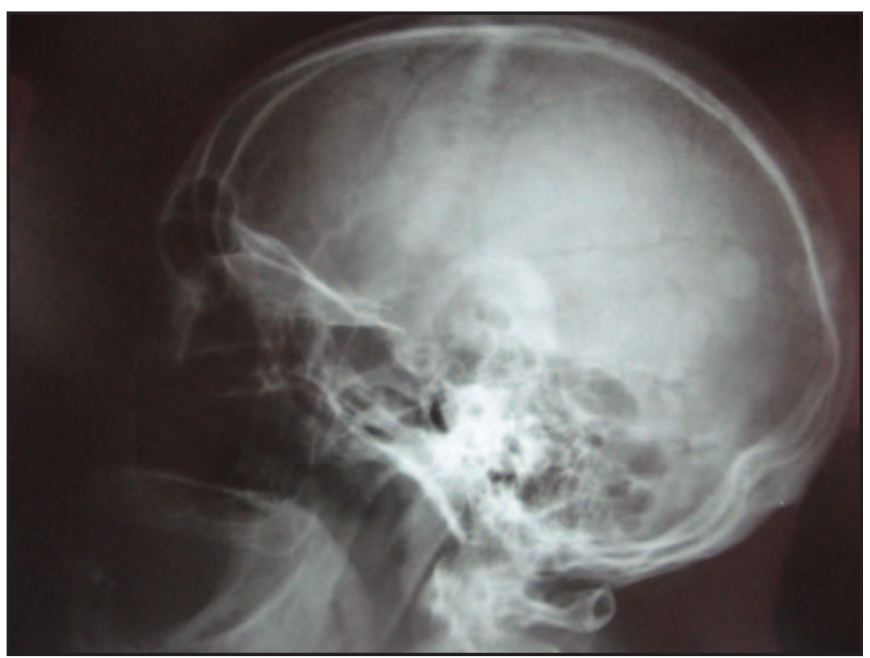

Fig. 5. Rx de cráneo: no se evidencian cambios.

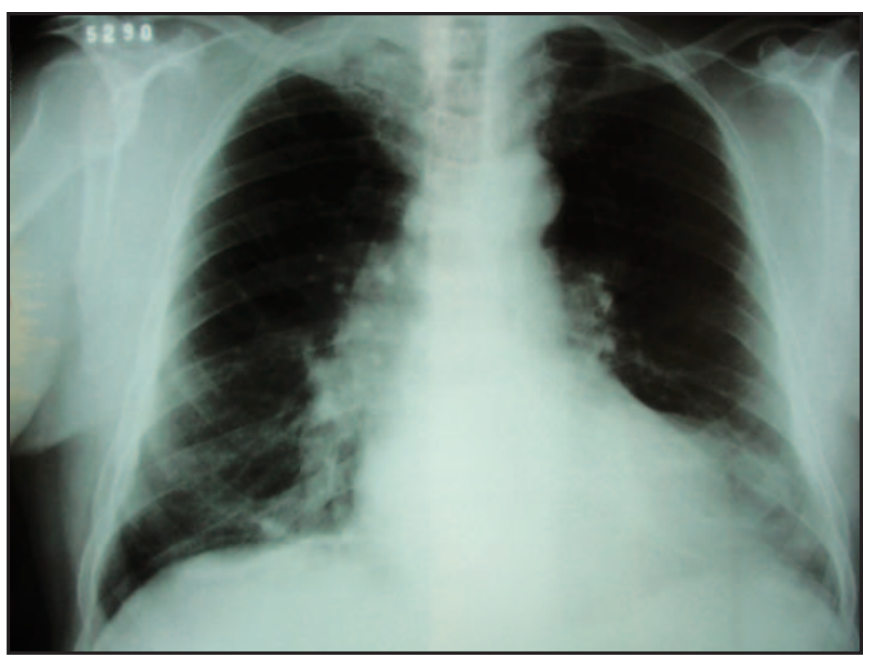

Fig. 6. Rx de Tórax: No se evidencian cambios.

tado los cuales podrían ser la señal de estar ante una patología tan compleja como es el plasmocitoma, he aquí la importancia de un diagnóstico temprano de parte del odontólogo tratante $(6,12)$. En el paciente que se reporta, se realizan las ayudas diagnósticas pertinentes lo que permitió llegar al diagnóstico correcto para así emplear la terapéutica adecuada.

De las diferentes opciones de tratamiento, la elección del tratamiento del paciente reportado en el artículo muestra que la radioterapia con un diagnóstico certero por medio del análisis histopatológico, se reflejará en la resolución temprana de la patología a 


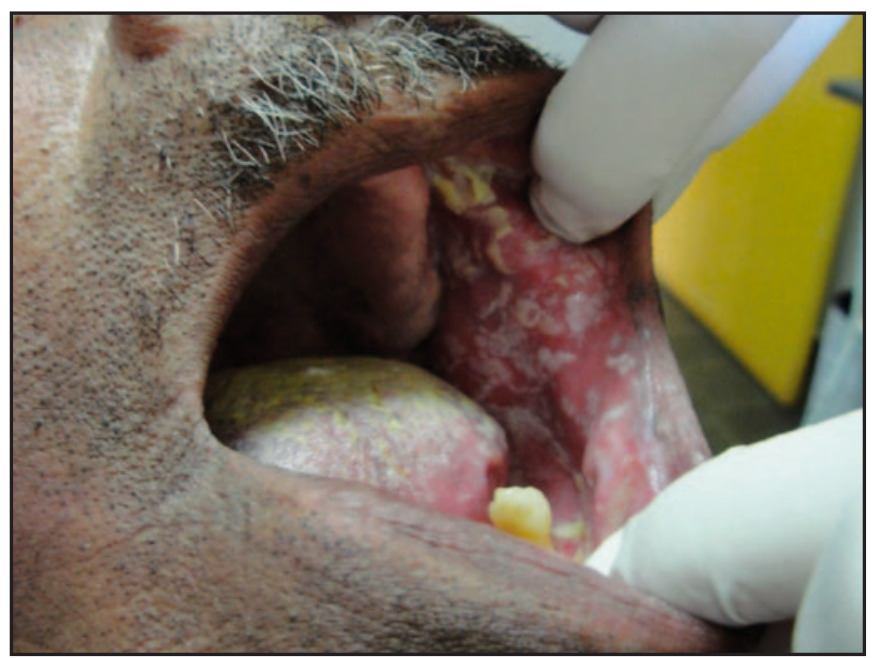

Fig. 7. Mucositis severa en mucosas yugal, labial y lengua.

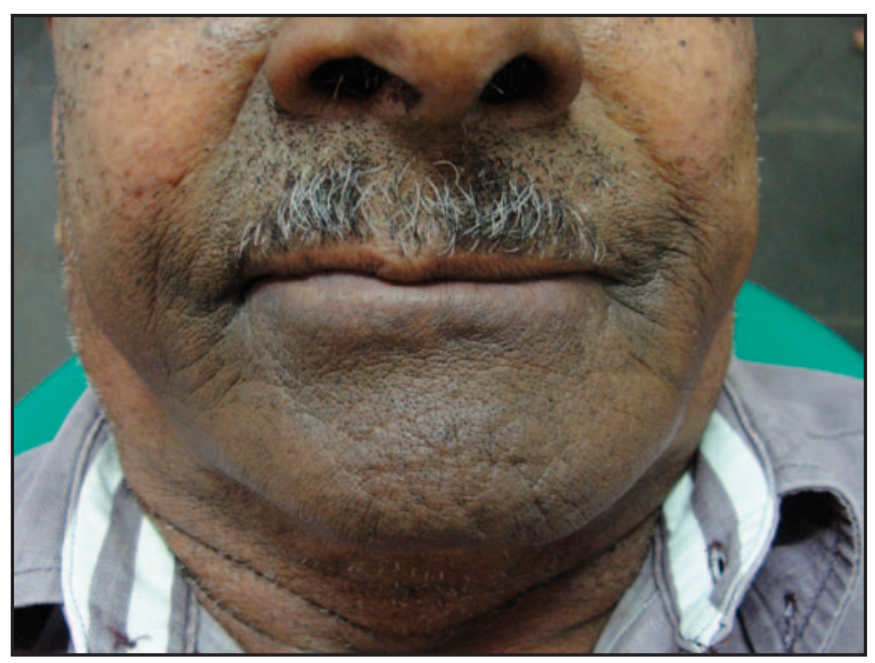

Fig. 8. Cambios en el color facial como resultado de la radioterapia.

la cual se está enfrentada. Es notorio que los resultados de laboratorio, los cuales incluyen pruebas bioquímicas, urianálisis y hematológicos así como estudios radiográficos de cráneo y tórax del paciente tratado, demuestran que la elección de un enfoque terapéutico con radioterapia, no debe ser empírico, sino por lo contrario, llenarse de razones biomédicas para lograr un resultado excelente $(2,7,9,16)$.

\section{BIBLIOGRAFÍA}

1. Damm DD. Mandibular radiolucency. Plasma cell neoplasm. Gen Dent 2009 Nov-Dec;57(6):672-5.
2. Elias HG, Scott J, Metheny L, Quereshy FA. Multiple myeloma presenting as mandibular illdefined radiolucent lesion with numb chin syndrome: a case report. J Oral Maxillofac Surg 2009;Sep;67(9):1991-6.

3. Lewis K, Thomas R, Grace R, Moffat C, Manjaly G, Howlett DC. Extramedullary plasmacytomas of the larynx and parapharyngeal space: imaging and pathologic features. Ear Nose Throat J 2007; Sep;86(9):567-9.

4. Tong DC, Leaper MR, Colquhoun AN, Rich AM. An unusual presentation of oropharyngeal mucosal plasmacytosis related to toothpaste. J Laryngol Otol. 2008; Oct;122(10):1112-4.

5. Solomon LW, Wein RO, Rosenwald I, Laver N. Plasma cell mucositis of the oral cavity: report of a case and review of the literature. Oral Surg Oral Med Oral Pathol Oral Radiol Endod 2008;Dec; 106(6):853-60.

6. Pinto LS, Campagnoli EB, Leon JE, Lopes MA, Jorge J. Maxillary lesion presenting as a first sign of multiple myeloma: case report. Med Oral Patol Oral Cir Bucal 2007; Sep;12(5):E344-7.

7. Moshref M, Mashhadi-Abbass F, Sargolzaei S, Nafarzadeh S. Extramedullary plasmacytoma of the gingiva. Arch Iran Med 2007 Jan;10(1):91-3.

8. Demetriades N, Prabhudev RK, Pokrovskaya N, Solomon LW, Shastri KA. A clinico-pathologic correlation. Extramedullary plasmacytoma. J Mass Dent Soc 2008;Fall;57(3):56-8.

9. Razaq S, Kessler HP, Phillips W. Oral and maxillofacial pathology case of the month. Solitary plasmacytoma of bone. Tex Dent J 2008; Feb;125(2):182-3, 8-90.

10. Sakai Y, Ikeya J, Morita I, Sato K. Extramedullary plasmacytoma of the tonsil diagnosed by fineneedle aspiration cytology. Ann Diagn Pathol 2008; Feb;12(1):41-3.

11. Canger EM, Celenk P, Alkan A, Gunhan O. Mandibular involvement of solitary plasmocytoma: a 
case report. Med Oral Patol Oral Cir Bucal 2007; Jan;12(1):E7-9.

12. Ozdemir R, Kayiran O, Oruc M, Karaaslan O, Kocer U, Ogun D. Plasmacytoma of the hard palate. J Craniofac Surg 2005;Jan;16(1):164-9.

13. Anil S. Solitary plasmacytoma of the maxilla-a case report and review of the literature. Gen Dent 2007;Jan-Feb;55(1):39-43.

14. Dores GM, Landgren O, McGlynn KA, Curtis RE, Linet MS, Devesa SS. Plasmacytoma of bone, extramedullary plasmacytoma, and multiple myeloma: incidence and survival in the United States, 1992-2004. Br J Haematol 2009;Jan; 144 (1):86-94.
15. Tseng JT, Cheng CJ, Lee WR, Wang KH. Plasma-cell cheilitis: successful treatment with intralesional injections of corticosteroids. Clin Exp Dermatol 2009 Mar; 34(2):174-7.

16. Millesi W, Enislidis G, Lindner A, Schobel G, Ewers $\mathrm{R}$, Drach J, et al. Solitary plasmocytoma of the mandible-a combined approach for treatment and reconstruction. Int $J$ Oral Maxillofac Surg 1997 Aug; 26(4):295-8.

\section{CORRESPONDENCIA}

Daniel Antunes Freitas

Correo electrónico:

danielmestradounincor@yahoo.com.br 